\title{
A LandSCAPE ANAlysis of Gene Flow AND POPULATION REDUCTIONS IN THE TIGER SALAMANDER (AMBYSTOMA TIGRINUM) ACROSS THE NORTHERN RANGe OF Yellowstone National PARK
}

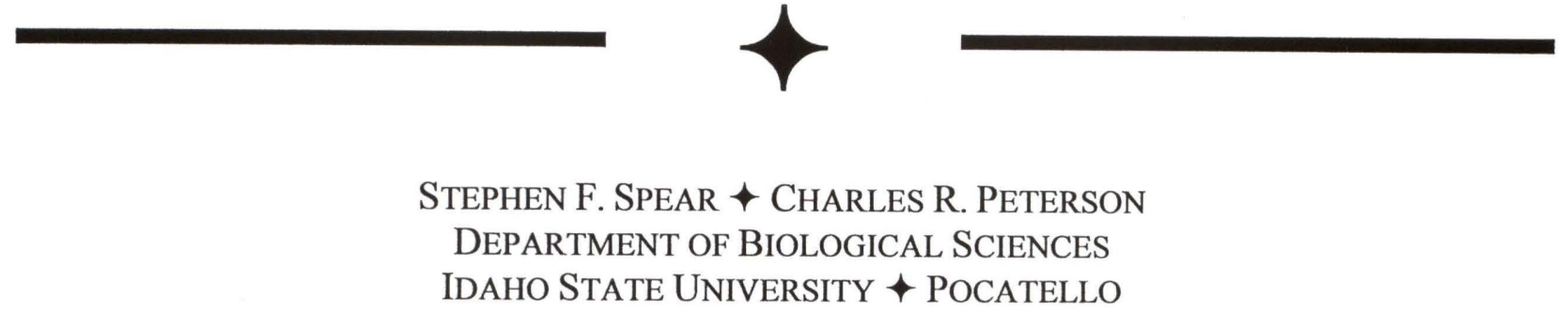

\section{$\downarrow \quad$ INTRODUCTION}

In the past two decades, there has been increasing awareness of a general pattern of decline of amphibian species (Semlitsch 2003a). A variety of factors have been implicated in these declines, including habitat alteration, climate change, chemical contamination, disease, invasive species, and commercial exploitation. It is likely that these different hypothesized causes combine to reduce populations. For example, human development such as roads or houses may not only destroy habitat, but also facilitate introduction of chemicals, invasive species, and disease. Habitat degradation is much easier to reverse or mitigate than the other factors (Semlitsch 2002). Therefore, protecting habitat from excessive human disturbance may indeed be the most productive way to conserve amphibian populations.

While declines have been documented across all amphibian groups, pond-breeding amphibians are a large group of amphibians that are especially vulnerable to habitat disturbances (Semlitsch 2003b). Species in this group require wetlands to breed, but in most cases spend the majority of the year in terrestrial habitats. Therefore, any type of freshwater aquatic or terrestrial habitat modification may impact populations. Furthermore, many of these species are more abundant in small, isolated wetlands that lack predators (i.e., fish). Isolated wetlands are the least protected of all wetland types and their loss eliminates breeding habitat, which can disrupt population dynamics. Even if the breeding habitat is protected, there are no current regulations that preserve the surrounding terrestrial habitat that amphibians use most of the year. Semlitsch and Bodie (2003) have proposed enacting terrestrial "buffer zones" to protect core terrestrial habitat, but this has not yet occurred.

There have been various solutions proposed to mitigate habitat disturbances, such as creating artificial wetlands or preserving as much favorable habitat as possible (Semlitsch 2002). However, evaluating the potential success of these methods requires knowledge of both the dispersal ability and habitat preferences of the species. For example, if a new wetland is created to replace a destroyed wetland, but the two areas are separated by habitat that deters amphibian movement, then the mitigation will be ineffective. Therefore, it would be useful to managers to develop models that can predict where amphibians will move based on the landscape.

One method would be to use variable genetic markers to estimate dispersal and structure among subpopulations. Estimates of gene flow across a fine spatial scale could provide a good indication of how much dispersal is occurring across the area. A recent study (Berry et al. 2004) tested the ability of genetic markers to accurately predict dispersal and found that the results from a three- 
month genetic study were the same as those from a seven-year mark recapture project. Once gene flow is estimated, then these results can be used to determine which habitats influence gene flow. This could indicate which types of habitat these animals most commonly use. Additionally, recent population reductions can be detected through the use of genetic markers. Therefore, this technique allows researchers to make inferences about several aspects of population structure in a much shorter time frame than using more direct field methods.

In this study, we examined the genetic structure and habitat associations of an ambystomid salamander, the Tiger Salamander (Ambystoma tigrinum), across the northern range of Yellowstone National Park. Tiger salamanders are the most widespread species of the mole salamander family. They are a classic pond breeding species in that most individuals only use wetlands for breeding and spend the rest of the year in terrestrial burrows. However, some individuals may become paedomorphic; that is, individuals become sexually mature while retaining larval characteristics. While this species is common throughout most of its range, there are several areas where different subspecies are of conservation concern, including populations in Washington and British Columbia (Garner et al. 2003), in Arizona (Semlitsch 2003a), and along the east coast (Buhlmann and Mitchell 2000).

The study area comprises most of the northern range of Yellowstone National Park and is characterized by mountainous terrain and mostly open sagebrush and other shrub habitat, with some sections of forested terrain. Three major rivers (including the Yellowstone) and many other smaller tributaries run through the area. However, overall, this is a dry area, with average annual precipitation of about 39 centimeters.

Several characteristics of this study area could have an influence on salamander abundance and gene flow. This region has been in a prolonged drought, with below average rainfall every since 1998 (Western Regional Climate Center data). This is especially important since Buhlmann and Mitchell (2000) speculated that droughts of greater than four years could be enough to cause large declines in a population of tiger salamanders in Virginia. However, it is currently unclear what effect this drought has had on amphibian populations in this area. Most of this landscape was completely covered by glaciers until 13,000-14,000 years ago (Pierce 1979). Therefore, this is the earliest date that the salamanders currently present could have colonized this area. Finally, disease has been found at several sites in this area due to ATV ranavirus (unpublished data), which might result in population declines.

This study had three main objectives: (1) Estimate the amount of gene flow among breeding ponds; (2) Determine if there have been any population reductions across the area; and (3) Use the genetic information to identify landscape and habitat variables that may be particularly important in influencing tiger salamander dispersal and population structure.

\section{$\uparrow \quad$ MeTHODS}

We surveyed ponds across the northern range for tiger salamanders during May-July of 2002 and 2003. Salamanders were captured using aquatic funnel traps and dipnetting techniques (Adams et al. 1997). Once a salamander was captured, we recorded its life stage and size, and then removed either a toe tip or a 1-centimeter portion off the end of the tail. We clipped toes for tissue samples in 2002, but switched to tail clips in 2003 because they tended to yield more DNA in extractions and also impacted the mobility of the organisms less (A. Storfer, personal communication). Toe and tail clips were stored in $100 \%$ ethanol in individually labeled vials after collection.

From mid-July to mid-August of 2003, we performed DNA extractions and polymerase chain reaction (PCR) amplifications in the Storfer laboratory at Washington State University. We did this work at WSU because the Storfer lab had previously developed primers for nine polymorphic microsatellite loci for use with tiger salamanders (Mech et al. 2003). We chose microsatellites as our genetic marker because they tend to be highly variable, which makes them ideal for detecting relatively recent patterns of gene flow (Jarne and Lagoda 1996). We used a standard phenolchloroform extraction protocol to isolate the DNA. We amplified the loci using the annealing temperatures described in Mech et al. (2003) on a programmable thermocycler, and ran PCR products on an agarose gel to check for successful amplifications. We amplified all nine loci in the collected samples. The Idaho State University Molecular Research Core Facility ran the samples on an ABI 377 (ABI Systems Inc.) sequencer. Because we had used fluorescent primers in the PCR reactions, we were able to multiplex and run 3 loci in each lane. After each run, the gel was analyzed and saved as a file using the program GENESCAN (ABI 
systems, Inc.). We then scored each locus for each sample using the program GENOTYPER (ABI systems, Inc.).

\section{Genetic analyses}

After scoring each sample, we used the program GENEPOP (Raymond and Rousset 1995), which allowed us to test for linkage disequilibrium and Hardy-Weinberg equilibrium among the loci and samples. Linkage disequilibrium occurs when genes are not in random association, and therefore each locus is not independent (Hartl and Clark 1997). It is also important to test for Hardy-Weinberg equilibrium for both loci and sample populations to identify whether they are under some selection or nonrandom mating occurs. Either of these events could violate assumptions of certain statistical analyses.

We used FSTAT 2.9.3. (Goudet 2001) to test for population differentiation based on individual genotypes present at a site. We then used ARLEQUIN (Schneider et al. 2000) to calculate $F_{\text {st }}$ and test for isolation by distance. The statistic $\mathrm{F}_{\text {st }}$ is a measure of the genetic differentiation between subpopulations and is based on allele frequencies, following the method proposed by Weir and Cockerham (1984). Isolation by distance states that with greater distance between populations there will be lower levels of gene flow (Slatkin 1993). We graphically tested for isolation by distance by converting $\mathrm{F}_{\text {st }}$ to $\mathrm{M}$, which is a direct estimate of gene flow presented by Slatkin (1993), and then graphed the $\log$ of $\mathrm{M}$ against the log of geographic distance. We also statistically tested for significant isolation by distance using the Mantel test (Mantel 1967).

We examined population declines using the program BOTTLENECK (Cornuet and Luikart 1996) to compare observed and expected heterozygosity to determine if there is evidence for a recent bottleneck in the population. Typically, a heterozygote excess would be expected in recently reduced populations. This excess is due to the fact that a recent reduction in population size reduces allelic variation more quickly than heterozygosity (Luikart et al. 1998), even though heterozygosity is ultimately over time. In this program, we used a two-phase mutation model (Di Rienzo et al. 1994) with multistep mutations accounting for either $5 \%$ or $10 \%$ of mutations, which is believed to be the most likely scenario for microsatellites. A Wilcoxon sign-rank test was used to determine which sites had significant heterozygote excesses. This test is most appropriate because it can achieve high statistical power if the average sample size per site is 30 or less.

\section{Analysis of landscape effects on gene flow}

For the analysis of the influence of landscape variables on among-site gene flow (measured by $\mathrm{F}_{\mathrm{st}}$ ), we used several different possible movement paths. The first two routes we used were a straight-line topographic distance between sites, and then a "stepping-stone" topographic route using salamander localities previously identified by the Idaho State University Herpetology Laboratory. In the "stepping-stone" model, an individual had to move to the nearest site before advancing through the landscape, with the eventual destination being one of the sampling sites. We considered the direct distance to be a null hypothesis to compare against the alternative courses. To create both these paths, we manually digitized each route using ArcGIS 8.2 (ESRI, Redlands, CA). We wanted to use the actual topographic distance along each route instead of the simpler linear distance. This required knowing the change in slope across the study area which we accomplished through several steps. First, because the study area encompassed several DEMs, we used ArcInfo workstation to mosaic the DEMs together to form one continuous layer. We then converted this combined DEM into a TIN layer, which converts the two-dimensional DEM into a three-dimensional layer. We used the TIN to determine a slope value for every $10 \times 10$ meter pixel across the study area, and then converted the 3D slope layer from the TIN into a feature shapefile layer, which allowed for intersection with digitized routes. We used the Xtools extension in ArcGIS to calculate the length of each route that was within each slope value, and finally used the cosine trigonometric function to convert linear distances to the actual topographic distance.

Along with this topographical distance, the landscape variables that we used to compare with genetic distance were mean wetland likelihood, percent of each cover type along the route, elevation, number of streams and rivers crossed by each route, and whether one or both of the sites in the pairwise comparison contained large larval forms. The wetland likelihood came from a GIS layer developed by Chris Wright at Montana State University. We adjusted the wetland likelihood values to be more biologically meaningful for salamanders by subtracting each value from 100 . Therefore, a high value in the adjusted grid would indicate lower wetland likelihood. We refer to this as "reverse wetland likelihood". To calculate a mean value for 
reverse wetland likelihood along the route, we again used the intersection tool and Xtools extension of ArcGIS to calculate the length of each route that went through each of the different values of reverse wetland likelihood, the same method we used for the slope. We calculated the percent of the total route within each value and then multiplied the proportion by the reverse wetland likelihood. For example, if $20 \%$ of a route had a reverse wetland likelihood of $50 \%$, we would multiply 0.2 times the wetland value of 50. We did this across all reverse wetland likelihood values and then summed the products. These calculations resulted in a single weighted mean of this variable for each route that was not dependent on the distance traveled. The next layer, cover type, was divided into 14 discrete categories, so to incorporate this factor into the analysis, we calculated the percentage of the route that lies in each category and used this directly, so that there are 14 cover type values for each route.

The other three variables were much simpler to quantify. To incorporate elevation, we used the difference between the elevation of the two sites, regardless of the topography in between. For stream and river crossing, the variable was the number of times the route crossed a stream or river, based on a stream shapefile developed for Yellowstone. Finally, to account for presence of potential paedomorphic individuals, we assigned 0 to a route if neither site had large larvae, a 1 if one site had large larvae, and 2 if both sites had large larvae. We included this variable because paedomorphic individuals cannot leave the breeding pond, and this might reduce gene flow.

While the combination of these variables includes most of the probable factors that would influence gene flow, it also creates a large number of different variables to incorporate into an analysis (up to 19). Therefore, even though the dependent variables are not independent points, we ran all the variables in a forward stepwise multiple regression in SAS. This approach allowed us to exclude variables $(\mathrm{P}>0.5)$ that did not explain any of the variation in genetic distance. We could then take the remaining variables that contributed to the $r^{2}$ and compare them with genetic distances using a partial Mantel test in FSTAT 2.9.3 (Goudet 2001). This test is a modification of multiple regression and accounts for the non-independence of points by creating a random distribution to use as a null distribution and test for significance. We used 10,000 randomizations to create this distribution. This test will give the partial correlations for each variable after controlling for any variables already entered into the model. For example, distance and number of river crossing across each route are correlated. Therefore, we always entered distance first into the model so that the correlation for the river variable would reflect the influence of river crossings and not distance. Because we had multiple comparisons for each model, we used a sequential Bonferroni correction (Sokal and Rohlf 1995) to determine the appropriate $P$ value based on an alpha of 0.05 .

Along with the straight-line distance and stepping-stone paths, we also wanted to test if the genetic data supported an alternative hypothesis of salamander movement based directly on the independent variables of slope, wetland likelihood, and the combination of these two factors. However, because each of these is based on fine resolution pixels, it would be too time consuming to manually digitize these routes. Instead, we created least cost paths using ArcInfo workstation. To do this, we first produced cost-distance grids for each sampling site based on the values of each raster grid (i.e. slope, reverse wetland likelihood). Once we created a costdistance surface for each variable, we were then able to use this surface to generate a least cost path between all sites for all variables. We created three least-cost paths between pairs of sites; two based on a single variable, and one with slope and wetland probability combined. To combine the different grids, we added them together in ArcInfo. This created a grid with the two values for each variable added together. We could then create a cost-distance surface based on this new grid, and then create the least cost path.

Once we had created these least cost paths, we converted them from grids to line shapefiles. We then performed the same regression and Mantel test analysis as with the straight-line and stepping stone routes, with one exception. Because we directly incorporated wetland probability into the least cost paths, we did not use it separately in the model along with the topographical distance. Therefore, for the least cost path routes, we used topographical distance, elevation, river crossings, cover type, and presence of large larvae as independent variables.

\section{RESULTS}

Of the ponds surveyed, we were able to get sufficient sample sizes $(>15)$ at ten sites. The intersite distances ranged from 500 meters to over 50 kilometers and sites were as far west as Gardiner, Montana and as far east as the Lamar Valley (Figure 1). We were able to use 199 individuals from the ten 
sites for my genetic analyses. For most of these samples, all of the microsatellite loci amplified in PCR. Eight of the nine loci used were polymorphic, and none of the eight loci showed any significant linkage. Furthermore, all loci were within HardyWeinberg expectations. All but one of the sampled pond populations was within Hardy-Weinberg equilibrium as well. The exception was Rainbow Lake, which had a significant heterozygote deficit. At this site, we found several dead large larval individuals, and tissue from these individuals contained ATV ranavirus DNA.

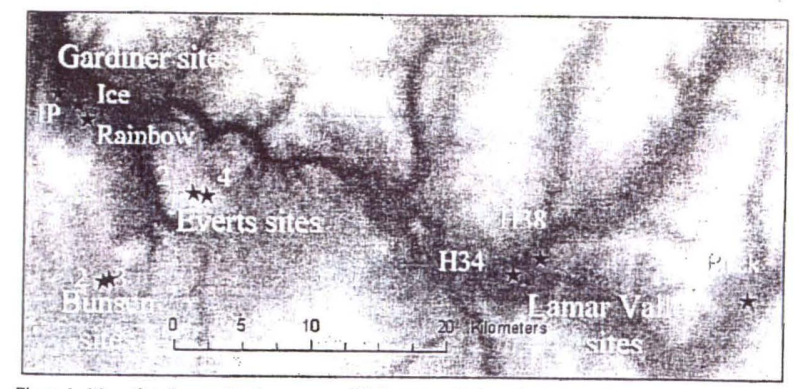

Figure 1. Map of study area (northern range of Yellowstone National Park with sites represented by stars. Names of each site are nearest to each star. Gardiner sites include IP, Ice, and Rainbow. The two Bunsen Valley sites. Sites and 3 and the two Everts sites are labeled 2 and 4. Sites H34, H38 and Buck are the Lamar designated by Hill and Rainbow and Buck are names appearing on topographic maps, H34 and H38 are names

\section{Gene Flow and Population Differentiation}

Overall, there was high genetic differentiation among sites across the study area (Table 1). In fact, the only sites that were not significantly differentiated from one another based on individual genotypes were the two Bunsen sites and also the two Everts sites. These were the only two pairs of sites that were less than a kilometer away from each other. As would be expected based on these results, the $\mathrm{F}_{\text {st }}$ value (0.24) was high across the study site. The individual pairwise comparisons for each site are included in Table 1. The $\mathrm{F}_{\text {st }}$ values showed higher differentiation among most pairwise comparisons, and in general, only near neighbors had values that were not statistically different. The only non-significant genetic distance that was greater than 10 kilometers was between the populations in Bunsen 2 and H38. The shortest geographic distances that yielded significant differences in $\mathrm{F}_{\text {st }}$ were 1.3 kilometers and 1.6 kilometers, which were between Ice Lake and IP, and Ice Lake and Rainbow Lake, respectively. These $\mathrm{F}_{\text {st }}$ results are primarily in agreement with the population differentiation tests performed in FSTAT that were based on individual genotypes. Along with significant differentiation, there was a significant isolation by distance pattern across the study area, based on the $F_{\text {st }}$ data, as evidenced both graphically (Figure 2) and by the Mantel's test $(\mathrm{P}=0.001)$.
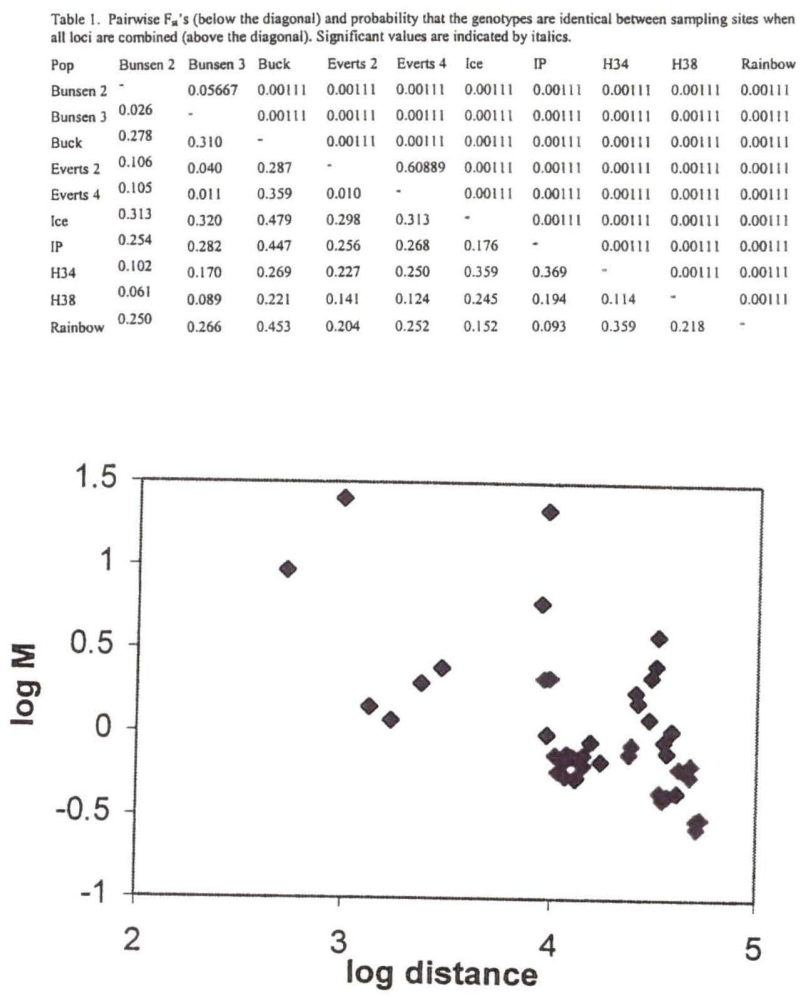

Figure 2. Comparison of gene flow (M) against geographical distance. Gene flow estimates are based on $\mathrm{F}_{\text {st }}$ values. Both $\mathrm{M}$ and distance are log transformed.

\section{Bottleneck results}

Based on the program BOTTLENECK, there is support for recent reductions in these populations. Results were slightly different dependent on which two-phase mutation model was used. The model with only $5 \%$ multistep mutations was more conservative. With this model, five of the ten sites showed significant heterozygote excess after standard Bonferroni correction. These populations were Bunsen $2(\mathrm{P}=0.00195)$, Bunsen $3(\mathrm{P}=0.00195)$, Everts $4(\mathrm{P}=0.00195)$, IP $(\mathrm{P}=0.00195)$, and $\mathrm{H} 34$ $(\mathrm{P}=0.00391)$. These same sites were also significant in the $10 \%$ multistep mutation model, along with an additional two populations including Everts 2 and Ice Lake, both of which had P-values of 0.00391 . The only ponds not to show any sign of a recent bottleneck were Buck Lake, H38, and Rainbow Lake.

\section{Gene flow and environmental variables}

Overall, distance was the most consistent predictor variable for each of the hypothetical routes (Table 2) and was the only variable included in every 
model. For the straight-line route (Figure 3), there were four variables included in the partial Mantel test based on the results of the multiple regression. These variables included topographical distance, elevation difference, open shrub habitat, and number of rivers and streams crossed. It should be noted that open shrub included fire regeneration areas and excludes areas dominated by sagebrush, which was its own separate category. All four of these variables were significant (Table 2), with distance explaining the most variation. Overall, the combined model had a $\mathrm{R}^{2}$ of 0.828 , which was the highest of any model. Distance and elevation were positively associated with $\mathrm{F}_{\mathrm{st}}$, whereas open shrub habitats and river crossings (after adjustment for distance by the test) were negatively associated.

\begin{tabular}{|c|c|c|c|c|c|c|}
\hline Route & Mantel test & P-value & Bonferroni value & Significance & $r$ & overall model $r^{2}$ \\
\hline \multirow[t]{4}{*}{ Straight-line } & $F_{z t} \cdot$ distance & 0.0002 & 0.0125 & - & 0.587 & \multirow[t]{4}{*}{0.828} \\
\hline & $\mathrm{F}_{\mathrm{s}} \cdot{ }^{*}$ elevation & 0.0044 & 0.0167 & - & 0.422 & \\
\hline & $F_{n}{ }^{*}$ open shrub & 0.008 & 0.025 & - & -0.397 & \\
\hline & $F_{x} \cdot$ rivers & 0.0096 & 0.05 & - & -0.384 & \\
\hline \multirow[t]{2}{*}{ stepping stone } & $\begin{array}{c}F_{\mathbf{u}^{*}} \text { distance } \\
\mathrm{Fst}^{\circ} \text { dry meadow }\end{array}$ & $\begin{array}{l}0.0003 \\
0.0004\end{array}$ & $\begin{array}{l}0.0167 \\
0.025\end{array}$ & : & $\begin{array}{r}0.495 \\
-0.491\end{array}$ & \multirow[t]{2}{*}{0.603} \\
\hline & $F_{n t} \cdot$ rev, wetlend & 0.0305 & 0.05 & - & 0.325 & \\
\hline \multirow[t]{2}{*}{ least slope path } & $\begin{array}{c}F_{z_{*}} \cdot \text { distance } \\
\text { Fst }\end{array}$ & $\begin{array}{l}0.0002 \\
0.0028\end{array}$ & $\begin{array}{c}0.01 \\
0.0125\end{array}$ & : & $\begin{array}{l}0.527 \\
0.441\end{array}$ & \multirow[t]{2}{*}{0.69} \\
\hline & $\begin{array}{l}\mathrm{F}_{\mathrm{z}} \cdot \text { "dry meadow } \\
\mathrm{Fst}^{\circ} \text { - closed forest } \\
\mathrm{Fst}^{*} \text { "rivers }\end{array}$ & $\begin{array}{l}0.0087 \\
0.1102 \\
0.431\end{array}$ & $\begin{array}{c}0.0167 \\
0.025 \\
\text { NA }\end{array}$ & $\begin{array}{l}\dot{1} \\
\text { NS }\end{array}$ & $\begin{array}{r}0.381 \\
0.24 \\
-0.121\end{array}$ & \\
\hline Wetland prob path & $\begin{array}{c}F_{\mathrm{z}} \cdot \text { rivers } \\
F_{\mathrm{st}} \cdot \text { distance } \\
\mathrm{Fst}^{*} \cdot \text { open shrub }\end{array}$ & $\begin{array}{l}0.0001 \\
0.0002 \\
0.0813\end{array}$ & $\begin{array}{c}0.0167 \\
0.025 \\
0.05\end{array}$ & $\dot{\dot{n}}$ & $\begin{array}{l}-0.557 \\
0.542 \\
-0.263\end{array}$ & 0.673 \\
\hline slope + wetland & $\begin{array}{c}F_{\mathrm{u}} \text { "distance } \\
\text { Fst } \\
\text { Fst " rivers } \\
\text { "large larvae }\end{array}$ & $\begin{array}{l}0.0007 \\
0.0008 \\
0.007\end{array}$ & $\begin{array}{c}0.0167 \\
0.025 \\
0.05\end{array}$ & : & $\begin{array}{c}0.53 \\
-0.49 \\
0.389\end{array}$ & 0.672 \\
\hline
\end{tabular}

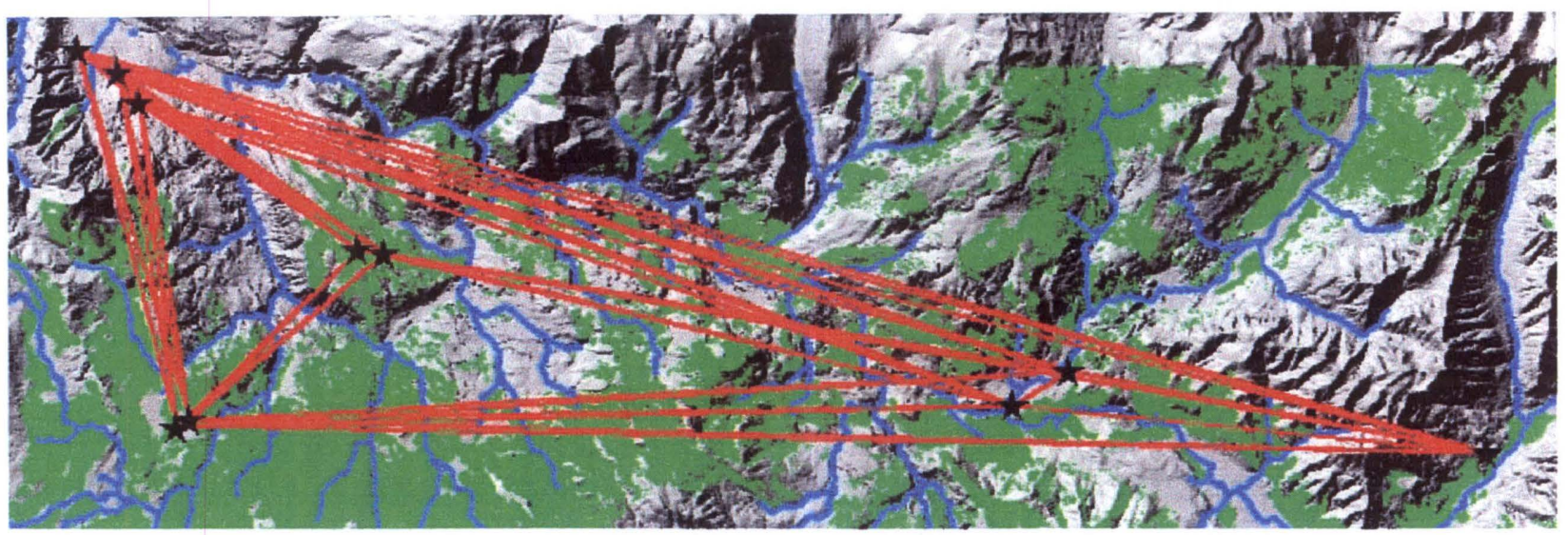

Figure 3. Map of direct route among sampling sites. Red lines indicate route and blue lines represent rivers and streams. Green areas on the map indicate areas with the open shrub/ fire regeneration cover type. Background is a shaded relief map of the study area.

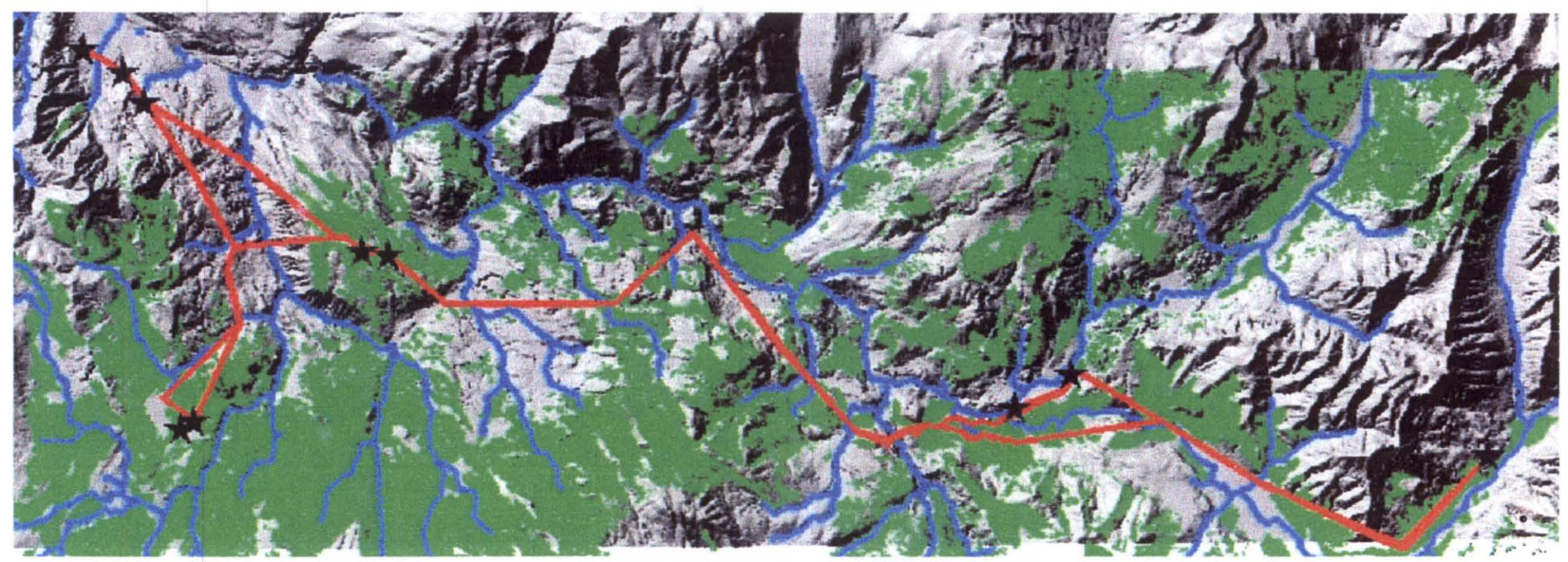

Figure 4. Map of stepping stone route among sampling sites. Red lines indicate route and blue lines represent rivers and streams. Green areas on the map indicate areas with the open shrub/ fire regeneration cover type. Background is a shaded relief map of the study area. 
The partial Mantel test based on a stepping stone route (Figure 4) included three variables in the model: topographical distance, dry meadow, and wetland likelihood. All of these variables were significant, with distance again explaining the most variation (Table 1). However, in this model, dry meadow and wetland likelihood were the significant predictors, with both being positively correlated with gene flow. This model only explained about $60 \%$ of the variance, which was much lower than that of the straight-line model.

The three least-cost paths generated in ArcInfo did appear to be better models than the stepping-stone approach, but still did not compare favorably to the straight-line model. Of the three, the least cost path (Figure 5) based on slope produced the highest $\mathrm{r}^{2}(0.69)$. This model, like the previous two, had distance as the most significant predictor of gene flow, with the presence of large larvae and dry meadow also being significant negative predictors of gene flow (Table 2). The latter result contrasts directly with the stepping stone model, which indicated dry meadows increase the rate of gene flow. This model also included closed forest habitat and rivers, but neither was significant. However, despite this lack of significance, removal of these two variables reduced the total $\mathrm{r}^{2}$ by about 0.2 , so we kept them in the model.

The route that was based on wetland likelihood (Figure 6) was the only one that did not have distance as the most significant predictor of $F_{\text {st }}$, although distance was a highly significant predictor. Instead, river crossings explained the highest amount of variance in the model. Open shrub was also included in this model, although it was not quite significant, but it explained enough of the variance that it warranted inclusion in the model. As in the straight-line model, both river crossings and open shrub were positively associated with increased gene flow and distance was negatively associated.

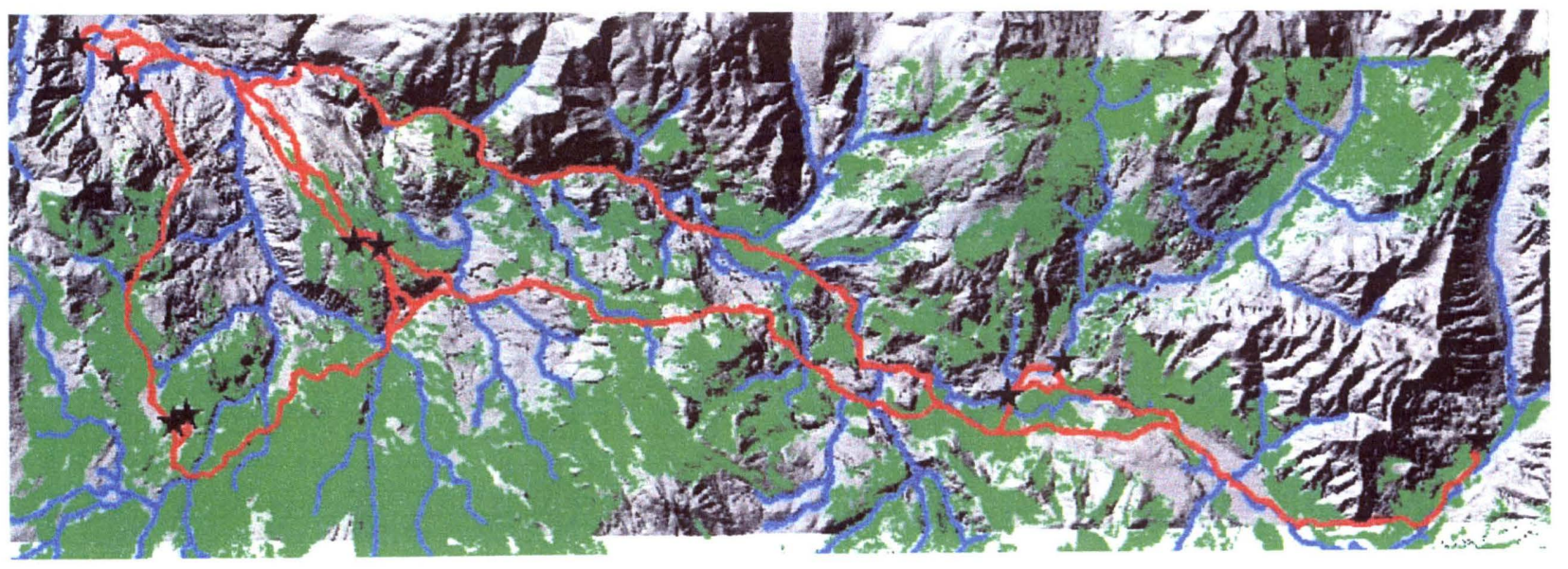

Figure 5. Map of least-cost path based on slope. Red lines indicate route and blue lines represent rivers and streams. Green areas on the map indicate areas with the open shrub/ fire regeneration cover type. Background is a shaded relief map of the study area.

Finally, the model based on the least cost path of both slope and wetland likelihood (Figure 7) accounted for the same amount of variance as the wetland likelihood model $(0.67$; Table 2$)$. This was the only model that did not include a habitat type variable. Instead, distance, river crossings, and presence of large larvae all contributed significantly to the model. Again, distance and presence of large larvae or paedomorphic individuals increased $\mathrm{F}_{\mathrm{st}}$, whereas river crossings decreased it.

\section{$\downarrow \quad$ DISCUSSION}

Overall, it appears that tiger salamanders across the northern range of Yellowstone are characterized by relatively low gene flow and high differentiation among sites. These results are consistent with an animal that has low vagility and a specific breeding center. The gene flow documented in this study is lower than most pond breeding amphibian studies (Newman and Squire 2001, Garner et al. 2003). There is good evidence for strong differentiation at a distance as fine as one kilometer, although this may be confounded by the more arid environmental conditions around the Gardiner sites. 


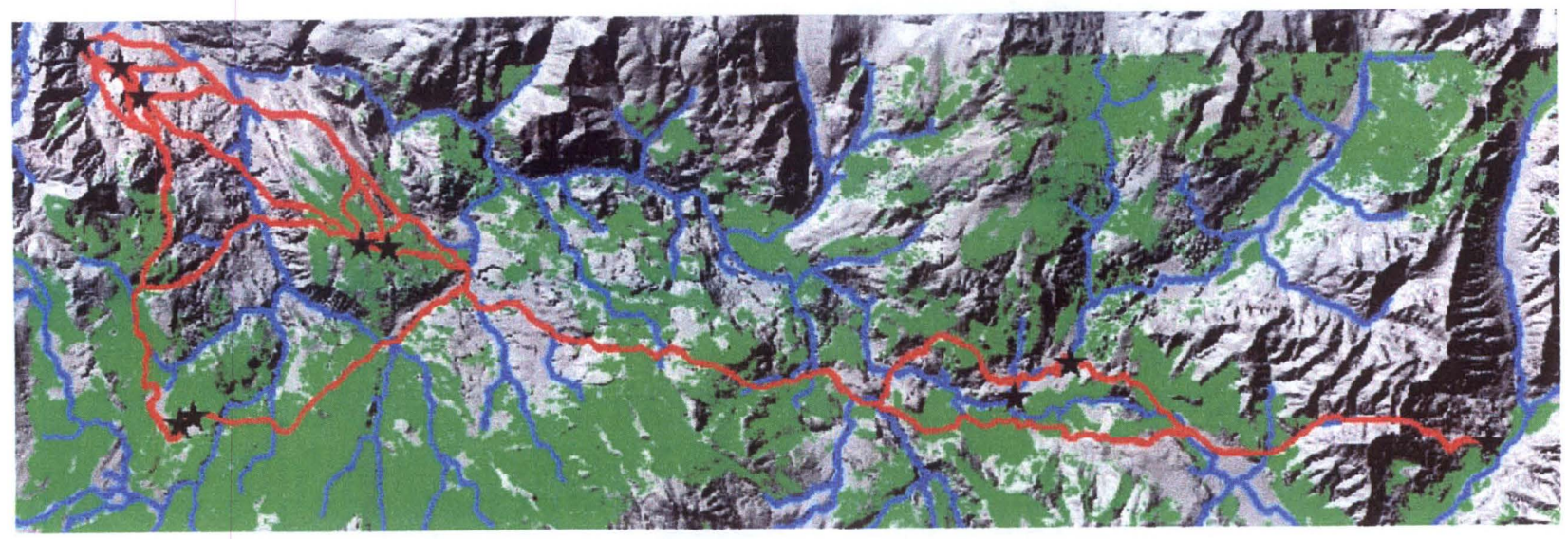

Figure 6. Map of least-cost path based on reverse wetland likelihood. Red lines indicate route and blue lines represent rivers and streams. Green areas on the map indicate areas with the open shrub/ fire regeneration cover type. Background is a shaded relief map of the study area.

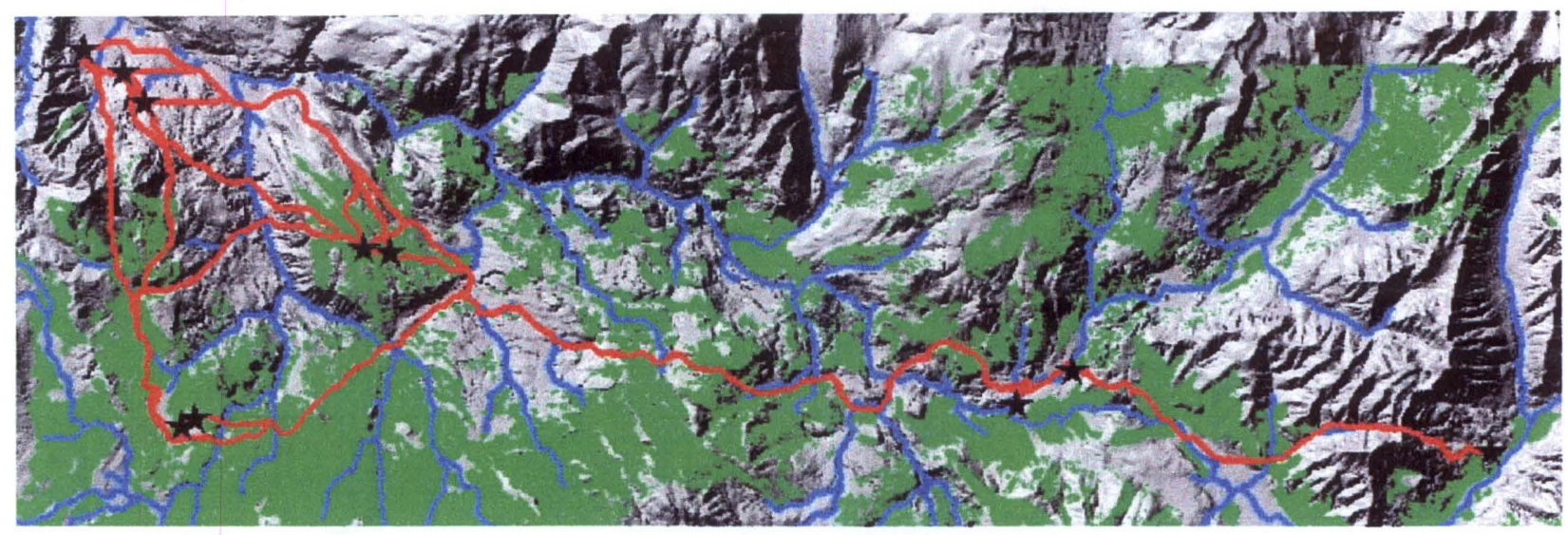

Figure 7. Map of least-cost path based on slope and reverse wetland likelihood. Red lines indicate route and blue lines represent rivers and streams. Green areas on the map indicate areas with the open shrub/ fire regeneration cover type. Background is a shaded relief map of the study area.

While there was overall isolation by distance across the northern range, there were some gene flow values that were both lower and higher than what would be expected by distance alone. Gene flow among the Gardiner sites and between H34 and H38 was lower than would be expected based on the close proximity of the sites to each other. All of these sites are surrounded by land that is characterized by an open sagebrush habitat. Numerous studies have shown that salamanders of the genus Ambystoma tend to avoid open habitats (Madison and Farrand 1998, deMaynadier and Hunter 1999, Rothermel and Semlitsch 2002).

Sagebrush habitats also tend to be drier than other types of habitat within the park.
Combined, these two factors could be a potential explanation for the low gene flow in this area. However, this hypothesis needs to be more rigorously tested before this conclusion can be made. The Gardiner sites are also characterized by the presence of large larvae, which may be either paedomorphic individuals or larvae overwintering. Based on Hill's results (1995), it is probable that some of these individuals are paedomorphic. Intuitively, it would seem that the presence of paedomorphic individuals would constrain gene flow due to the limited dispersal ability of these individuals. Interestingly, Routman (1993) found that there was no significant difference in gene flow between sites that had paedomorphic salamanders and between those sites that had 
transforming adults. While it is unclear exactly what the effect of paedomorphism is in this area, the $F_{\text {st }}$ value is higher on average between the Gardiner sites than between $\mathrm{H} 34$ and H38. This provides some support for paedomorphism decreasing gene flow, but this also needs further testing.

The gene flow results that were higher than expected involved the salamanders at the Bunsen sites. Specifically, Bunsen 3 showed high gene flow between both the Everts sites and Bunsen 2 showed relatively high gene flow with H38. The H38 result is peculiar because the distance between the two ponds is over 35 kilometers. Therefore, this result is likely due to either historical associations or homoplasy. Given the high differentiation across the area, the cause is probably not historical associations, but rather homoplasy. The more fascinating comparison is between Bunsen 3 and the Everts ponds. These sites are separated by about nine kilometers. This is certainly far longer than any documented oneway tiger salamander movement, but it may be possible to have gene flow between these sites over several generations using intermediate ponds as "stepping stones". Additionally, the Gardner River runs nearby both these sites and could form at least a one-way connection with salamanders traveling from Bunsen to Mt. Everts. It is unknown whether either of these hypothetical scenarios is likely. It is doubtful that the Gardner River is a connection considering that a small stream directly connects Ice Lake and Rainbow Lake, two sites that appear to be differentiated. Even if current gene flow is not occurring, the high gene flow values suggest that the same large population of salamanders colonized the Bunsen and Everts sites.

Not only does it seem that tiger salamanders are relatively isolated across the study area, it also appears that they have undergone population reductions in several sites across the northern range. BOTTLENECK detected significant heterzygote excesses, characteristic of population reductions, in 5-7 of the sites, depending on the mutation model used. These sites spanned most of the study area, and only the large ponds did not have such a reduction. The fact that only large ponds did not show bottlenecks indicates that drier conditions in recent years may have reduced populations. For example, during the early 1990s in the Slough Creek/Lamar River area, Hill and Moore (1994) found numerous ponds with tiger salamanders. These ponds were also within close proximity of each other. In the two years (2002-03) that we surveyed this area, most of these wetlands were dry or at very low levels that dried before any successful metamorphosis could take place. This reduces breeding success and might certainly lead to population reductions, especially if the salamanders have a metapopulation structure and rely on recruitment from more than one pond. This would explain the reduction in $\mathrm{H} 34$, which is in the Slough Creek area. There is no baseline data available for the Everts and Bunsen area, but especially in the Bunsen area, the wetlands appeared significantly reduced in size from what they would be in a wet year.

Another possible explanation for the population reduction is that disease has reduced populations in this area. We confirmed ranavirus in specimens from Rainbow Lake and also from Slide Lake, a pond between Mammoth and Gardiner, which were not included in this study due to small sample size. Salamanders were abundant at this site in the early 1990s (C. Peterson, personal communication), so disease may have affected that population. However, Rainbow Lake did not have significant heterozygote excess. Instead, it had a significant heterozygote deficit and was not in Hardy-Weinberg equilibrium. If this is due to disease, then this deficit could occur if disease had reduced the population so that the remaining survivors started to interbreed. This scenario requires that disease has been present in the population for some time, which may or may not be the case, as Rainbow Lake has never been regularly sampled. As far as the other populations that showed a bottleneck are concerned, there is no evidence that disease has contributed to these presumed declines. The only other confirmed ranavirus cases in tiger salamanders in the northern range were found at Rainey Lake, which is near Tower Junction and about five kilometers from the Slough Creek area. We did not survey this site because it was too shallow and drying. However, other than this site, there has not been suspected ranavirus cases near any of the other sites sampled in this area. Therefore, we would conclude that drought conditions and drying ponds are causing the population reductions in my sample sites.

\section{Landscape effects on gene flow}

The strong performance of the straight-line path was an unexpected result of this study. Based on the metapopulation potential for these organisms (Marsh and Trenham 2001, Semlitsch 2003b), a stepping-stone model would be anticipated. Similarly, wetland likelihood should affect the gene 
flow of an amphibian, and it has been shown that slope (specifically ridges) can serve as deterrents to gene flow (Funk 2004). While these other models still explained a great deal of the variation in the genetic data $(60-70 \%)$, they did not come close to the straight-line model (83\%). There are several possible explanations for this. The first, and simplest, is that the salamanders are moving in a relatively straight line. We have located tiger salamanders on steep slopes when an easier path from the pond was not far away. Even if the salamanders tend to move in a straight line, it is still unlikely that there could be direct gene flow between sites several kilometers away, which is longer than these salamanders could be expected to move in a single trip. The main problem with the stepping stone model may have been that there are sites that salamanders use that have not been identified and therefore were not included. However, the northern range of the park has been extensively surveyed for amphibians in the past ten years, reducing the possibility of missed sites. Another problem is that many of the sites used in the stepping stone model may be unsuitable for salamander use due to drought and disease. Several of the sites where salamanders have been observed were dry in 2002 and 2003 . Additionally, two of the sites included as stepping stones have had recent disease outbreaks, and are believed to have declining populations (Patla and Peterson 2004). Given that there has been one empirical study on a tiger salamander species that showed high movement between suitable ponds (Ambystoma californiense; Trenham et al. 2001), the lack of a stepping stone pattern suggests that these populations are becoming more and more isolated.

The factors that were significant predictors of gene flow include distance, elevation, river and stream crossings, open shrub habitat, dry meadow habitat and large larvae. The correlations of these variables were similar across the different models, with the one exception of dry meadow habitat, which was a positive predictor of gene flow in the stepping stone model and a negative predictor in the least slope model. Dry meadow should be expected to decrease gene flow. In fact, there has been no study to our knowledge that has concluded that dry meadow habitat would increase salamander movement. The fact that this was a positive predictor is another reason to doubt the validity of the stepping stone model.

While we expected distance to decrease gene flow, we did not expect open shrub and river crossings to increase it. Open shrub areas will have less cover and rivers and streams with fish were hypothesized barriers. Despite less overall cover, however, the shrubs could provide sufficient cover to allow for movement in a small animal like a salamander. One encouraging aspect of this result is that the open shrub habitat category included fire regeneration areas. Therefore, recently burned areas may not be a hindrance to movement. In addition, open areas may provide more space for more mammal burrows, which are an important habitat feature for tiger salamanders.

The effect of rivers and streams on gene flow could be due to the ongoing drought in the area. If isolated pond sites are becoming dry, then individuals that travel near riverine systems may be less likely to desiccate. This would certainly leave them more vulnerable to fish predation and it is unlikely that salamanders use the actual streams, but many of these areas have small pools adjacent to the stream that can provide habitat, and possibly a breeding area.

Finally, the tendency for elevation and presence of large larvae to decrease gene flow appears to be due primarily to the Gardiner sites, which are at the lowest elevation and the only sampled sites with paedomorphic individuals. It is difficult to say if either of these two variables are the primary reason for the strong differentiation of individuals at these sites. However, based on the straight-line model, it appears the large elevation difference has separated these three sites from all other sites, and the absence of open shrub among the three sites accounts for the high $F_{\text {st }}$ values between these sites. This explanation would also be in agreement with Routman's (1993) finding that the presence of paedomorphosis does not increase differentiation.

Overall, this study is consistent with the Funk (2004) hypothesis that mountain ridges are strong deterrents to amphibian gene flow based on the apparent genetic isolation of the low elevation Gardiner sites. However, the low $\mathrm{F}_{\text {st }}$ between the Bunsen and Everts sites, which have two major mountain ridges between them, counters this hypothesis. This study does not support previous work on other amphibians that showed that rivers are barriers and is the first study to indicate that an open shrub and fire regeneration habitat might be favorable to Tiger Salamanders. 


\section{Conclusions and Conservation Implications}

The low gene flow and apparent recent reduction in numbers of salamanders across the study area indicate that these subpopulations are becoming isolated and that declines are occurring across the area. This combination would decrease the likelihood that individual sites that go locally extinct would be "rescued" by adjacent populations. However, it appears that the presence of rivers and open shrub/fire regeneration habitats might counteract this isolation. It may be that the downfall from burned areas may provide substrate cover for this species. This could be especially important for areas that have lower density of small mammal burrows. The idea that fire improves tiger salamander gene flow is intriguing and deserves further investigation.

The lack of baseline genetic data make it impossible to state for certain that these populations are at immediate risk. These populations may have always had low gene flow, and recent reductions may be part of normal population fluctuations, which has been documented for amphibians (Semlitsch et al. 1996). However these results should provide warning that even though the Tiger Salamander has been considered stable across the northern range (Koch and Peterson 1995), this may not be the case. Future work in this area should focus on determining salamander abundance at sites from year to year, and also monitoring known breeding sites for signs of successful breeding and disease.

\section{$\downarrow \quad$ ACKNOWLEDGMENTS}

This project would not have been possible without the funding provided by the University of Wyoming - National Park Service Research Station.

We also received financial support from the Idaho State University Department of Biological Sciences and the ISU Graduate Student Research and Scholarship Committee. Additionally, we would like to thank Christie Hendrix and the National Park Service for research permits and logistical support for this project. Debra Patla aided us in selecting sampling sites for Tiger Salamanders.

We are especially grateful to Andrew Storfer and his laboratory at Washington State University for assistance with developing this project and allowing us to do most of the genetic work in his laboratory. Marjorie Matocq provided valuable help in analyzing the genetic data. Chris Wright generously allowed us use of his wetland likelihood layer, which is part of his dissertation research. We would also like to acknowledge Bob Klaver, Paul Bartelt, and Jeremy Shive, all of whom provided much assistance in doing the landscape analyses. Finally, we would like to thank Denim Jochimsen for her help and support throughout this project.

\section{Literature Cited}

Adams, M.J., K.O. Richter, and W.P. Leonard. 1997. Surveying and monitoring amphibians using aquatic funnel traps. Pp. 47-54 In Sampling Amphibians in Lentic Habitats. Edited by D.H. Olson, W.P. Leonard, and R.B. Bury. Olympia, WA. Society for Northwestern Vertebrate Biology.

Berry, O., M.D. Tocher, and S.D. Sarre. 2004. Can assignment tests dispersal? Molecular Ecology 13: 551-562

Buhlmann K.A. and J.C. Mitchell. 2000. Age of adult Eastern Tiger Salamanders (Ambystoma tigrinum tigrinum) in a Virginia sinkhole pond complex: Implications for conservation. The Journal of the Elisha Mitchell Scientific Society 116: 239-244.

Cornuet J.M. and Luikart G., 1996 Description and power analysis of two tests for detecting recent population bottlenecks from allele frequency data. Genetics 144:2001-2014.

DeMaynadier, P.G. and M.L. Hunter, Jr. 1999. Forest canopy closure and juvenile emigration by pond-breeding amphibians in Maine. Journal of Wildlife Management 63: 441-450.

Di Rienzo, A., A.C. Peterson, J.C.Garza, A.M. Valdes, M. Slatkin, and N.B. Freimer. 1994. Mutational processes of simple sequence repeat loci in human populations. Proceedings of the National Academy of Sciences 91: 3166-3170. 
Funk, WC. 2004. Patterns and consequences of dispersal in Columbia spotted frogs (Rana luteiventris). Dissertation. University of Montana, Missoula. 141 p.

Garner, T.W.J., S. Angelone, P.B. Pearman. 2003. Genetic depletion in Swiss populations of Rana latastei: conservation implications. Biological Conservation 114: 371-376.

Goudet, J. 2001. FSTAT, a program to estimate and test gene diversities and fixation indices (version 2.9.3). Available from http://www.unil.ch/izea/softwares/fstat.ht ml. Updated from Goudet (1995)

Hartl, D.L. and A.G. Clark. 1997. Principles of Population Genetics, $3^{\text {rd }}$ edition. Sinauer Associates, Sunderland, MA. 542 p.

Hill, S.R. 1995. Description of migratory chronology of adult tiger salamanders (Ambystoma tigrinum) and survey of larvae of the tiger salamander in the northern range of Yellowstone National Park. Master's thesis, Montana State University, Bozeman, Montana.

Hill, S.R. and R.E. Moore. 1994. Herpetological survey in the northern range of Yellowstone National Park. Annual Report to Yellowstone National Park. February 1, 1994. $21 \mathrm{pp}$.

Pierce, K.L. 1979. History and Dynamics of Glaciation in the Northern Yellowstone National Park Area. United States Geological Survey Professional Paper 729-F.

Raymond, M. and F. Rousset. 1995. GENEPOP (Version 1.2): Population genetics software for exact tests and ecumenicism. Journal of Heredity 86: 248-249.

Rothermel, B.B. and R.D. Semlitsch. 2002. An experimental investigation of landscape resistance of forest versus old-field habitats to emigrating juvenile amphibians. Conservation Biology 16: 1324-1332.
Routman, E. 1993. Population structure and genetic diversity of metamorphic and paedomorphic populations of the tiger salamander, Ambystoma tigrinum. Journal of Evolutionary Biology 6:329-357.

Schneider, S., D. Roessli, and L. Excoffier. 2000. ARLEQUIN Version 2.0001: a Software for Population Genetics Data Analysis. Genetics and Biometry Laboratory, University of Geneva, Switzerland.

Semlitsch, R.D. 2002. Critical elements for biologically based recovery plans of aquatic-breeding amphibians. Conservation Biology 16:619-629.

Semlitsch, R.D. 2003a. Introduction: General Threats to Amphibians. In R.D. Semlitsch (ed) Amphibian Conservation. pp1-7. Smithsonian Institution, Washington, D.C.

Semlitsch, R.D. 2003b. Conservation of PondBreeding Amphibians. In R.D. Semlitsch (ed.) Amphibian Conservaton. pp 8-23. Smithsonian Institution, Washington D.C.

Semlitsch, R.D. and J.R. Bodie. 2003. Biological criteria for buffer zones around wetlands and riparian habitats for amphibians and reptiles. Conservation Biology 17: 12191228.

Semlitsch, R.D., D.E. Scott, J.H.K. Pechmann, and J.W. Gibbons. 1996. Structure and dynamics of an amphibian community: Evidence from a 16-year study of a natural pond. In M.L. Cody and J.A. Smallwood (eds.) Long-term Studies of Vertebrate Communities. Pp 217-248. Academic Press, San Diego, CA.

Slatkin, M. 1993. Isolation by distance in equilibrium and non-equilibrium populations. Evolution 47:264-279.

Sokal, R.R. and F.J. Rohlf. 1995. Biometry, $3^{\text {rd }}$ edition. W.H. Freeman and Company, New York. 887 p. 
Trenham, P.C., W.D. Koenig, and H.B. Shaffer. 2001. Spatially autocorrelated demography and interpond dispersal in the salamander Ambystoma californiense. Ecology 82: 3519-3530.
Weir, B.S. and C.C. Cockerham. 1984. Estimating F-statistics for the analysis of population structure. Evolution 38: 1358-1370. 